\title{
Protocol Followed for Management of Hypernatremia in a White-tailed Deer Fawn (Odocoileus virginianus)
}

\author{
Nichole Rosenhagen ${ }^{1}$ and Julia K. Whittington, DVM ${ }^{2}$ \\ ${ }_{1}^{1}$ University of Illinois, College of Veterinary Medicine, Urbana, Illinois \\ ${ }^{2}$ University of Illinois, Department of Veterinary Clinical Medicine, Urbana, Illinois
}

\begin{abstract}
A white-tailed deer fawn with suspected trauma presented to a wildlife medical facility in central Illinois. Initial triage revealed clinical signs highly indicative of head trauma and shock, including ataxia, disorientation, and tachycardia. The fawn was administered subcutaneous isotonic fluids and oral meloxicam. The following day, hematologic evaluation revealed profound hypernatremia and azotemia. Meloxicam was discontinued and treatment with slow intravenous administration of 5 percent dextrose in water (D5W) was initiated to correct the hypovolemia and hypernatremia. Within three days, the fawn was able to suckle and ambulate normally. Chemistry values returned to normal within one week of admission. Electrolyte disturbances are common in orphaned neonatal animals suffering from starvation, dehydration, and shock, and inappropriate fluid therapy in the face of these disturbances may further compromise an animal's condition. Thus, the fluid composition, rate, and route of administration are critical for care of these patients.
\end{abstract}

Keywords: hypernatremia, white-tailed deer, Odocotleus virginianus, fluid therapy, electrolyte.

\section{INTRODUCTION}

Electrolytes are ions present in all bodily fluids that are closely regulated to achieve internal homeostasis. Potassium, calcium, phosphorus, and magnesium are found in highest concentrations within intracellular fluid, while sodium and chloride are maintained predominantly in extracellular fluid (Bohn 2012). Water intake and output, food consumption, and hormone signaling help keep these electrolytes within a narrow range for continued survival. Despite this ongoing balancing act, electrolyte abnormalities are common in wildlife that present to rehabilitation centers. Often, these imbalances are not grossly apparent as the body has the means to compensate for changes. But in extreme cases, the animal's compensatory ability is exceeded and clinical signs become evident.

Nichole Rosenhagen is a fourth year veterinary student at the University of Illinois College of Veterinary Medicine and student manager of the Wildlife Medical Clinic.

Julia Whittington, DVM, is a clinical associate professor of zoological medicine in the Department of Veterinary Clinical Medicine at the University of Illinois, College of Veterinary Medicine. She is the director of the $U$ of I Wildlife Medical Clinic.
Electrolyte disturbances arise secondary to a wide array of circumstances, including starvation, fluid loss from vomiting and/or diarrhea, lack of water intake, and organ dysfunction (Wellman et al). Because of the many roles electrolytes have in the body, an animal with an imbalance may present with clinical signs indicating pathology in any organ system. It is the examiner's job to utilize a patient's history and clinical presentation to interpret these abnormalities in order to effectively provide care for the animal. This paper describes the presentation, diagnosis, and treatment of severe hypernatremia (hyper-elevated, natremia-sodium, in the blood) in a white-tailed deer fawn (Odocoileus virginianus) presented for care to a wildlife rehabilitation facility.

\section{CASE REPORT}

A $3.4 \mathrm{~kg}$ neonatal female, white-tailed deer was presented to the Wildlife Medical Clinic at the University of Illinois after a member of the public found her alone in his yard. On presentation, the fawn was quiet and responsive, but ataxic and unable to stand without assistance. A cursory physical exam was performed to minimize handling and stress. The fawn's mucous membranes were dry, her extremities were cool, and her heart rate was greater than 200 beats per minute (ref. 90-110 bpm for neonatal calves), supporting a diagnosis of hypovolemia secondary to severe (10\%) dehydration (Smith 2002). No external signs of trauma were noted, but based on the brief exam and history, head trauma remained a differential diagnosis. Subcutaneous isotonic fluids (Lactated Ringer's injection [LRS], Hospira, Inc., Lake Forest, IL) were administered as well as a $0.2 \mathrm{mg} / \mathrm{kg}$ oral dose of meloxicam (Metacam ${ }^{\circledR}$, Boehringer Ingelheim, Vetmedica, Inc., St. Joseph, MO) to reduce cerebral inflammation associated with the suspected trauma. The patient was placed in an oxygen chamber and allowed to rest quietly for several hours. 
Once the fawn was more stable, a blood sample was collected for glucose and electrolyte evaluation. The data was compared to in-house reference ranges of healthy white-tailed deer fawns (unpublished data, Wildlife Medical Clinic, University of Illinois, College of Veterinary Medicine, Urbana, IL). Hypoglycemia was ruled out as the cause of the neurologic signs (174 $\mathrm{mg} / \mathrm{dL}$; ref. $60-105 \mathrm{mg} / \mathrm{dL}$ ), but the serum sodium was significantly increased $(206.6 \mathrm{mmol} / \mathrm{L}$; ref. $145-155 \mathrm{mmol} / \mathrm{L})$. Additionally, blood urea nitrogen (BUN) was increased (141 mg/dL; ref. 6-34 mg/dL), as was serum creatinine $(6.6 \mathrm{mg} / \mathrm{dL}$; ref. $0.8-1.2 \mathrm{mg} /$ $\mathrm{dL})$. A urine sample was not collected to assess specific gravity, but based on the physical exam findings, the azotemia was suspected to be pre-renal due to dehydration. A 20 ga catheter was placed in the left cephalic vein and slow fluid replacement therapy was initiated with five percent dextrose in water (D5W, 5\% Dextrose Injection, Hospira, Inc., Lake Forest, IL) administered intravenously (IV) at $15 \mathrm{ml} / \mathrm{hr}(106 \mathrm{ml} /$ $\mathrm{kg} /$ day) to correct the hypovolemia and severe sodium imbalance. Standard fluid rate to provide maintenance fluids $(100 \mathrm{ml} / \mathrm{kg} /$ day $)$ and correct 10 percent dehydration in a neonatal fawn would be up to 200 $\mathrm{ml} / \mathrm{kg} /$ day.

The next morning, the fawn was still ataxic but displayed improved mental awareness. The fawn was started on frequent, small volume feedings of milk replacer. A second blood sample after 48 hours of fluid therapy revealed that the fawn's serum sodium level had decreased to $179 \mathrm{mmol} / \mathrm{L}$; BUN and serum creatinine were still increased and she was estimated to be 8 percent dehydrated based on blood chemistry values and the presence of tacky mucous membranes. She weighed $3.8 \mathrm{~kg}$. The catheter in the cephalic vein was no longer patent, and a new 20 ga catheter was placed in the right jugular vein. The fluid composition was changed to 1:1 D5W and LRS and administered at $23 \mathrm{ml} / \mathrm{hr}(145 \mathrm{ml} / \mathrm{kg} /$ day $)$. After 72 hours of care, the fawn's weight was $4.2 \mathrm{~kg}$; she was able to stand and ambulate without assistance and was estimated to be 7 percent dehydrated. The fluid rate was increased to $30 \mathrm{ml} / \mathrm{hr}(171 \mathrm{ml} / \mathrm{kg} /$ day $)$ and she was administered 20 percent mannitol (Mannitol Injection 20\%, Neogen, Corp, Lexington, KY) at $2 \mathrm{~g} / \mathrm{kg}$ IV to promote diuresis. The mannitol dose was repeated every six hours for a total of three doses. After the first two doses, the fluid rate was increased to $46 \mathrm{ml} / \mathrm{hr}$ $(263 \mathrm{ml} / \mathrm{kg} /$ day; 1.5 times maintenance + dehydration rate) for several hours to facilitate renal perfusion and replace the fluid lost in urine. Within four days of intake, the fawn's neurologic signs had resolved. She weighed $4.3 \mathrm{~kg}$ and was eating and drinking without assistance. Her IV fluids were changed from the mixed solution to full strength LRS and decreased to $20 \mathrm{ml} /$ $\mathrm{hr}(111 \mathrm{ml} / \mathrm{kg} /$ day $)$. By day six, all blood chemistry values were within normal limits and the fawn was exhibiting age-appropriate behavior. Fluid therapy was discontinued and the fawn was monitored for another four days before being transferred to a rehabilitation center for continued care until release.

\section{DISCUSSION}

Sodium ions are critical to maintaining plasma osmolarity. Biologically, plasma osmolarity refers to the number of osmoles of glucose, sodium, potassium, and urea per liter of blood plasma. The proper balance of these components is critical for maintaining normal fluid volumes and cell function in the body. Changes in osmolarity can cause cells to shrink or swell and subsequently become damaged or die. Sodium pumps in cell membranes maintain sodium concentration differences across cell membranes; however, sodium moves freely between the vascular and interstitial spaces to maintain blood volume and blood pressure (Bohn 2012). The causes of fluctuation in sodium concentration can be direct or indirect. Direct causes, with few exceptions, reflect excessive sodium ingestion. Indirectly, sodium concentration is affected by water volume within the body (Spromberg 2009). In wildlife, the most common cause of sodium disturbance is severe dehydration, resulting in hypovolemic hypernatremia. A decrease in water consumption, an increase in free water loss, or both, will cause an increase in the concentration of blood sodium. As sodium levels climb, an animal's thirst mechanism is triggered and antidiuretic hormone is released to minimize free water loss in urine. Additionally, intracellular volume decreases as fluid moves out of the cells to compensate for the lost extracellular water. Once plasma sodium levels reach a critically high level (170 $\mathrm{mEq} / \mathrm{L}$ in most species), the body can no longer compensate and clinical signs manifest (Spromberg 2009). The signs associated with hypernatremia are predominately associated with changes in the central nervous system, including ataxia, tremors, disorientation, lethargy, and blindness. In cases of severe hypernatremia, the brain offsets the volume depletion by generating idiogenic osmoles, osmotically active solutes that act as sponges to pull water into the brain and maintain its hydration (DiBartola 2012).

When managing these patients, the intensity of treatment varies with both the severity of the hypernatremia, as well as its rate of onset. In acute or moderate cases, a rapid decrease in sodium levels with proper fluid administration corrects the problem 


\begin{tabular}{|c|c|c|c|c|c|}
\hline $\begin{array}{l}\stackrel{亠}{ \pm} \\
\stackrel{c}{0} \\
\end{array}$ & & 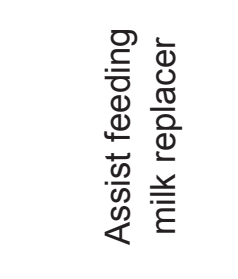 & 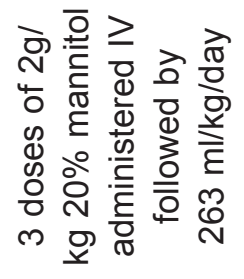 & 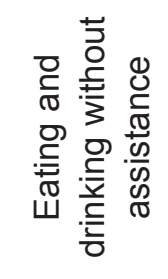 & \\
\hline 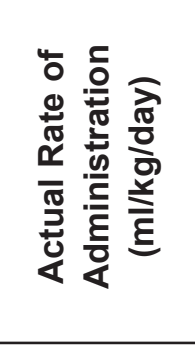 & $\stackrel{8}{\circ}$ & $\stackrel{L}{\stackrel{f}{+}}$ & 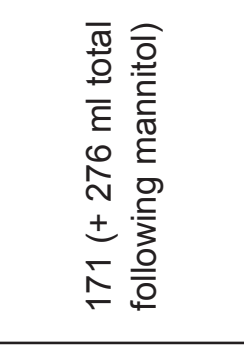 & $\underset{\mp}{\mp}$ & 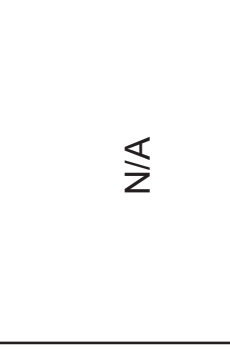 \\
\hline 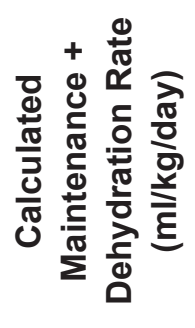 & ঃ & $\stackrel{\infty}{\leftarrow}$ & $\stackrel{R}{\stackrel{2}{r}}$ & $\stackrel{\stackrel{\sim}{N}}{\sim}$ & $\stackrel{8}{\circ}$ \\
\hline 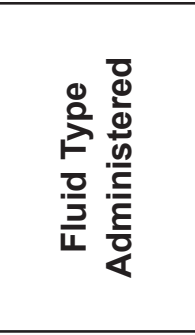 & 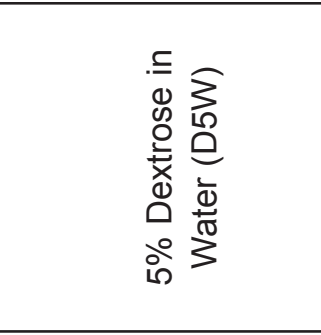 & 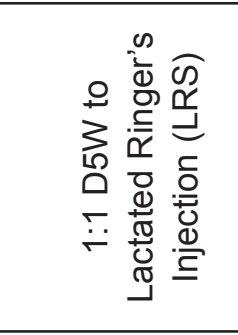 & 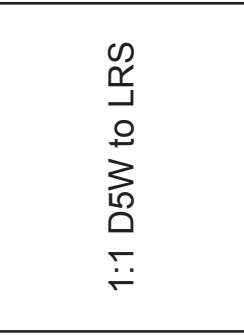 & 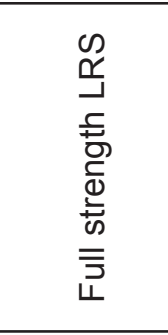 & 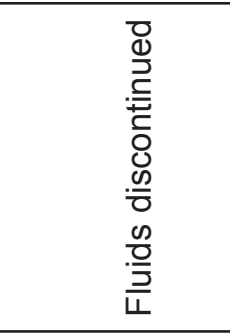 \\
\hline 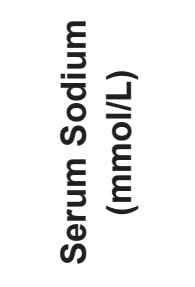 & $\begin{array}{l}0 \\
\ddot{Q} \\
\stackrel{N}{ }\end{array}$ & $\stackrel{g}{\stackrel{R}{\leftarrow}}$ & 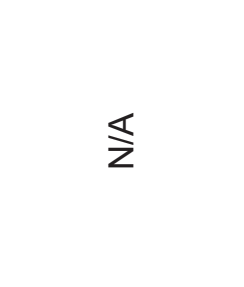 & $\stackrel{5}{\leftarrow}$ & $\stackrel{\sim}{\underset{\sim}{\sim}}$ \\
\hline 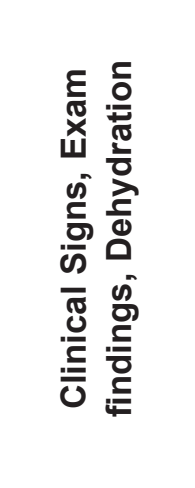 & 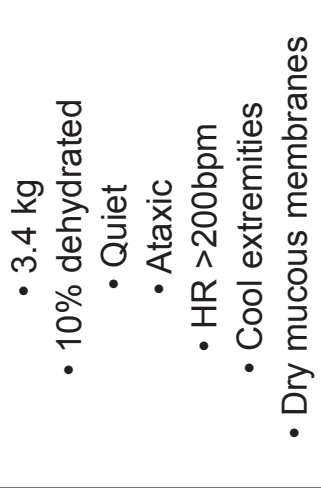 & 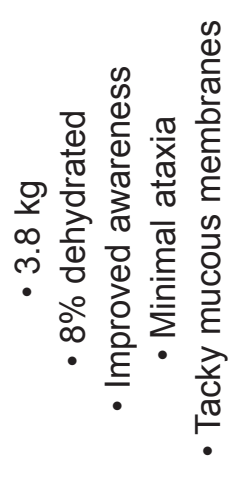 & 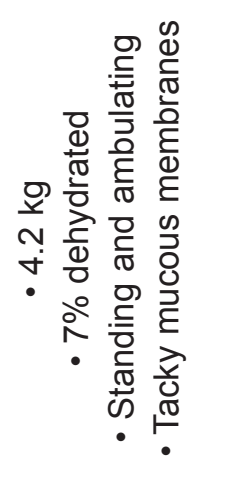 & 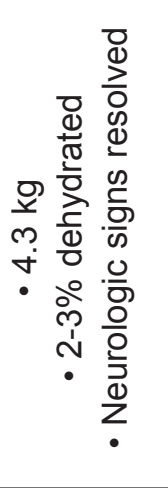 & 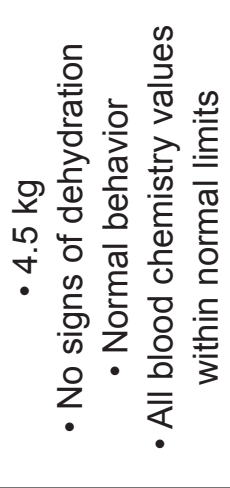 \\
\hline 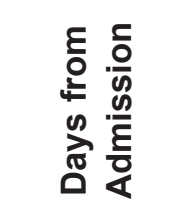 & 0 & $\sim$ & $m$ & $\nabla$ & 0 \\
\hline
\end{tabular}


without further complication (Devey 2009). In severe or chronic cases however, plasma sodium levels must be lowered slowly, not exceeding a decrease of more than $0.5 \mathrm{mEq} / \mathrm{L} / \mathrm{hr}$ for $48-72$ hours (Spromberg 2009). Additionally, free choice water should not be provided; instead, the patient should be offered small amounts of water frequently throughout the treatment period until serum sodium levels normalize. Rapid administration of fluids to decrease the sodium level is contraindicated as the resultant large fluid uptake by the idiogenic osmoles in the brain leads to cerebral edema and iatrogenic damage in the central nervous system (DiBartola 2003). The rate of fluid administrated in the aforementioned case, while lower than what might be provided for a patient with normal serum sodium levels, may be more aggressive than other patients can safely tolerate. The fawn in this case report was monitored regularly for signs of cerebral edema or fluid overload, and the information gleaned from her clinical signs, heart and lung auscultation, and blood chemistry values dictated her specific protocol. It is important to tailor a treatment program to the individual patient, not to the disease, and adjust fluid rates as needed based on the animal's clinical appearance.

In cases of hypovolemic hypernatremia, where the etiology is loss of free water rather than a true increase in sodium, the most pressing treatment requirement is to return the animal to a euvolemic state (DiBartola 2012). Several described methods exist to reach such a state. Despite the various fluid composition options, any fluid provided must be given directly into the vasculature to be effectively absorbed. This can be done via intravenous or intraosseous administration. Dextrose in free water, as described in this case, can be used to treat hypernatremia caused by free water deficit. When administered intravenously, dextrose in free water quickly becomes hypotonic, leading to a fluid influx into the volume depleted cells (DiBartola 2003). Though successful, this treatment option must be planned carefully and monitored for the duration of the treatment to prevent cell swelling or the described cerebral edema. If the hypernatremia is a result of hypotonic fluid loss rather than free water deficit, the hypovolemia is likely to be more critical and an isotonic fluid solution should be utilized to rapidly restore extravascular volume (DiBartola 2003). A final treatment approach focuses on matching fluid sodium levels to those in the body via intravenous administration of hypertonic saline. Over time, the fluid sodium concentration should be decreased to allow a controlled decrease of serum sodium.
Electrolytes are utilized by every organ in the body, and their concentrations are often interconnected, so a deficiency of one may lead to a deficiency or an excess of another. This universal effect, as well as the interdependence of electrolytes, makes it challenging to diagnose an electrolyte abnormality based on an animal's clinical presentation alone. The most efficient method to identify an imbalance is with a blood chemistry analyzer; however, when advanced diagnostic testing is unavailable, there are a number of basic tests that can provide some insight to the animal's electrolyte levels. For increases in serum sodium specifically, the urine is concentrated and the specific gravity is elevated. Additionally, in cases of indirect hypernatremia, the patient likely will show signs of dehydration and hypovolemia. These include prolonged skin tent, delayed capillary refill time, increased packed cell volume (PCV), cold extremities, sunken eyes, increased heart rate and lethargy or obtunded mentation. If the hypernatremia is secondary to an excess of salt in the diet, the animal may show signs of hypervolemia as the increased total sodium leads to water retention.

When considering an electrolyte abnormality in a patient, it is imperative that the examiner remember to investigate other causes of clinical signs. For hypernatremia and the corresponding neurologic signs, the differential list is extensive. Without a reliable serum sodium value, it is important to rule out some of the more common causes of neurologic deficits before beginning treatment.

Electrolyte imbalances are common in wild animals that present to rehabilitation centers. In extreme situations, they can be life-threatening. Fortunately, these disturbances often can be treated. Proper and thorough assessment of these cases is crucial to their management and success, and with the right tools and information, these animals can make a full recovery.

\section{LITERATURE CITED}

Bohn, A. A. 2012. Laboratory Evaluation of Electrolytes. Veterinary Hematology and Clinical Chemistry, 2nd edition (M. A. Thrall, G. Weiser, R. W. Allison, and T. W. Campbell, editors). Wiley-Blackwell: Ames, IA. Pp. 378-392.

Devey, J. J. 2009. What's All the Salt About? Understanding Sodium, Chloride and Potassium. Proceedings of the American Board of Veterinary Practitioners: Austin, TX.

DiBartola, S. P. 2003. Disorders of Sodium: Hypernatremia and Hyponatremia. Proceedings of the Atlantic Coast Veterinary Conference: Columbus, $\mathrm{OH}$. 
DiBartola, S. P. 2012. Disorders of Sodium.

Proceedings of the Atlantic Coast Veterinary

Conference: Columbus, $\mathrm{OH}$.

Smith, B. P. 2002. Chapter 17: Initial Management and Physical Examination of the Neonate. Large Animal Internal Medicine. St. Louis, MO.

Spromberg, L. 2009. Electrolyte Imbalances:

Warning Signs and Fluid Choices. Proceedings of the International Veterinary Emergency and Critical Care Symposium: Seattle, WA.

Wellman, M. L., C. W. Kohn and S. P. DiBartola. 2012. Chapter 1: Applied Physiology of Body Fluids in Dogs and Cats. Fluid, Electrolyte, and Acid-base Disorders in Small Animal Practice. St. Louis, MO. (N.

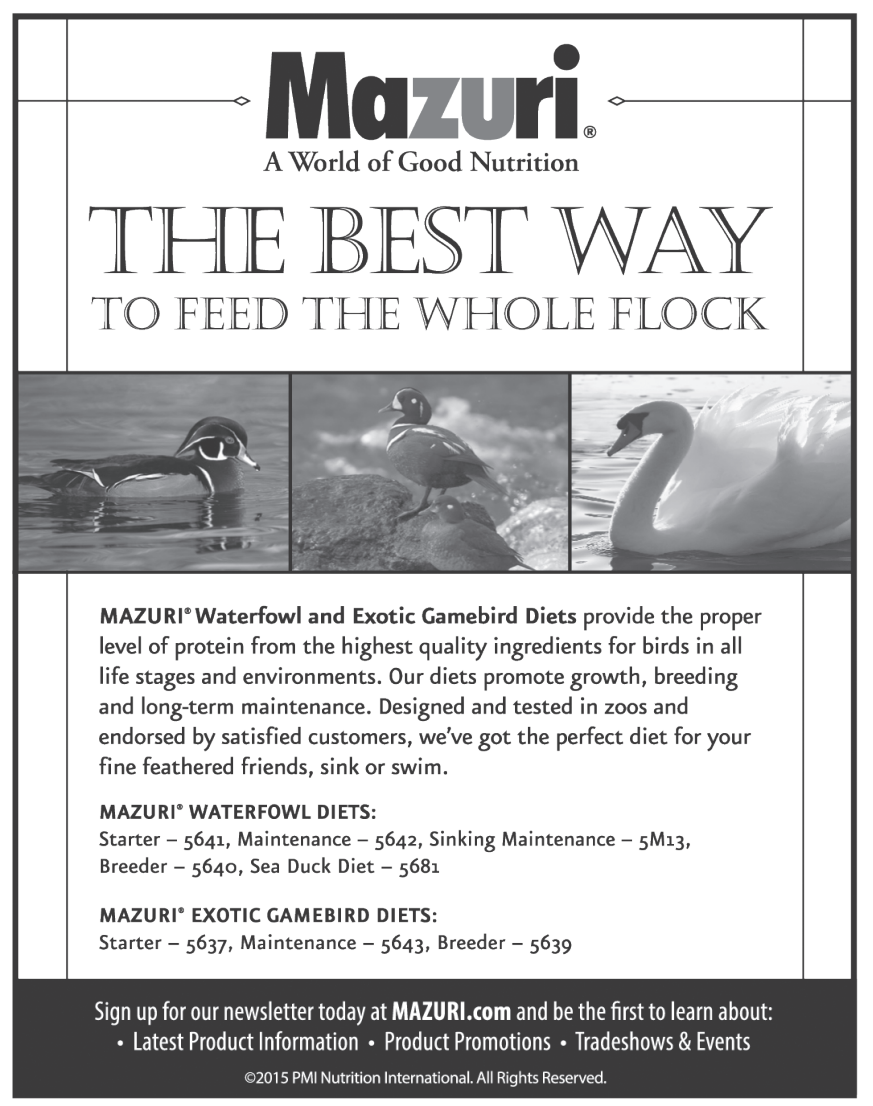

$\left(\begin{array}{l}\text { W R } \\ \text { A }\end{array}\right) \begin{aligned} & \text { National } \\ & \text { WildLIFE Rehabilitators } \\ & \text { Association }\end{aligned}$

Introducing the NWRA Polar Fleece!

Featuring:

- Full-length zipper

- Zippered pockets

- NWRA logo
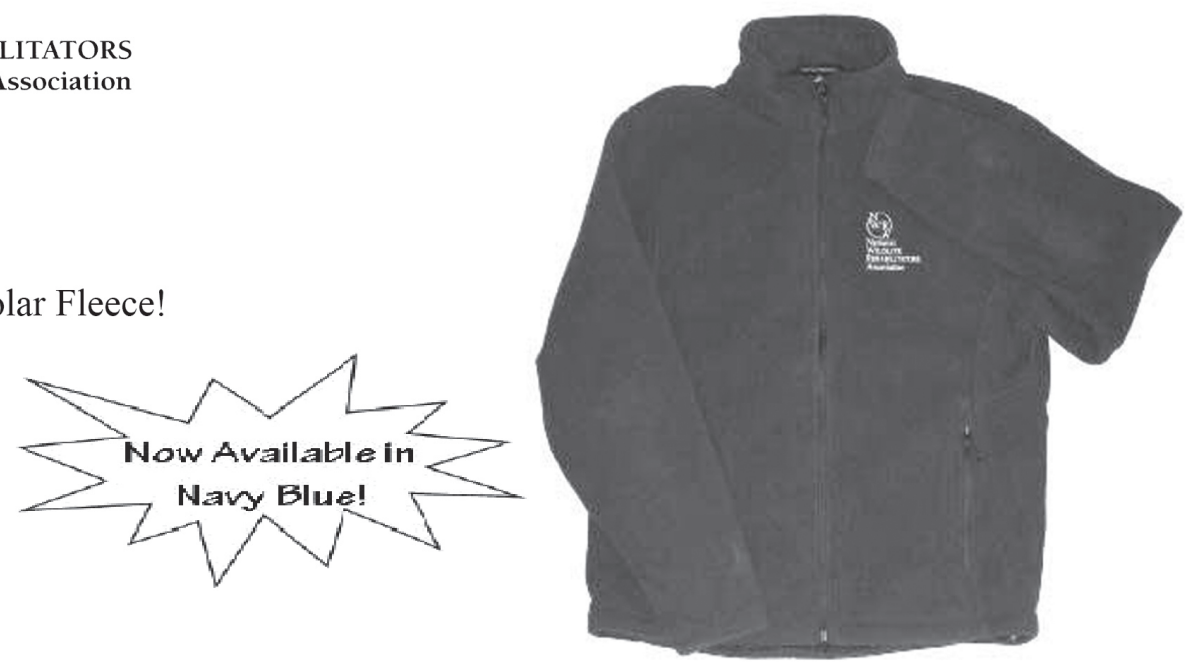

The NWRA Polar Fleece is available in forest green or navy blue, sizes S-XXXL, for $\$ 55.75$ (p\&h included) for members, and $\$ 58.75$ ( $\mathrm{p} \& \mathrm{~h}$ included) for non-members. For XXL, add $\$ 2.00$, and for XXXL, add $\$ 3.00$. For shipping outside the US, please call 320-230-9920 for pricing.

To order your NWRA Polar Fleece, visit our website at <www.NWRAwildlife.org $>$

or send a check or money order in US Dollars to:

2625 Clearwater Rd, Suite 110, St. Cloud, MN 56301 